\title{
Performance Analysis for Channel Utilization in Wireless LAN
}

\author{
Shweta Singh \\ KIET Group of Institutions
}

\author{
Naresh Chandra \\ KIET Group of Institutions
}

\author{
Arun Kumar Tripathi \\ KIET Group of Institutions
}

\begin{abstract}
Wireless network plays an important role in field of communication. Now a days people are always eager to access the data at any time of moment. The wireless network allows to access the Internet from anywhere at any time. The connectivity can be easily established via devices such as smartphones, laptops, etc. this paper simulates a campus WLAN using OPNET IT GURU EDUCATIONAL VERSION 14.5. Depending on certain justified scenarios the evaluation has been done onto stations participating in the network. Also, performance of the network is measured on parameters such as congestion, delay, throughput, Traffic, Load in wireless environment. The paper discusses about how to handle the channel performance issues using different access mechanisms.
\end{abstract}

\section{Keywords}

Wireless LAN, TCP Connection, Congestion, Delay, Throughput, Traffic, Load, RTS, CTS, Retransmission, Media Access Delay, CSMA/CA, NAV.

\section{INTRODUCTION}

Wireless network is based on IEEE 802.11[1] standard and evolved in late nineties. The deployment of wireless technology changed the thinking of the generation, leading the people to access the information onto the network. This provides the facility to bring closer to the "anything, anytime, anywhere" promise of mobile networking.

One of the requirements of the IEEE 802.11 standard is to handle the devices with the concept of mobility i.e. the devices as mobile stations in Wireless Local Area Network (WLAN). Mobile stations are devices that can easily make use of WLAN [2] in motion. IEEE802.11 standard was made to accomplish the services that were commonly found in wired networks such as throughput, reliable data delivery, and continuous network connections. This standard performs at Medium Access Control (MAC) [3] sublayer and three PHY (physical) layers.

Using the two underlined classes of MAC technique, to implement the structure of network; Point Co-ordination Function (PCF): co-ordinates the network channel and Distributed Co-ordination Function (DCF): employs CSMA/CA [4] technique. Both these classes utilize the virtual carrier-sense mechanism by means of Network Allocation Vector (NAV) in which each station can predict the future traffic onto the medium.

Point Co-ordination Function: It is a MAC technique in which priority is being provided to the channel access mechanism. It is located above the DCF and hence enhances the DCF through a new co-ordination function, called Hybrid Co-ordination Function (HCF).

The Access Point (AP) in the network acts as Point Coordinator (PC) that manages the channel access using poling mechanism, to determine which station is to initiate the transmission.

> Distributed Co-ordination Function: It is a fundamental technique of IEEE 802.11 based WLAN standard which employs CSMA/CA technique with binary exponential back-off algorithm.

Every station, in this mechanism, is allowed to sense the medium to transmit and defers to sense till medium is available. It facilitates the communication through short Request-to-Send / Clear-to-Send RTS/CTS [5] frames including positive ACK for successful delivery to the destination device. This does not solve the hidden/exposed terminal problem.

The configurations [6] supported by WLAN can be categorized as follows:

A) Ad-hoc Network: The network is said to be ad-hoc as it does not rely on existing infrastructure, such as routers in managed wireless networks. Under this, all devices consists equal status on a network.

B) Infrastructure BSS: BSS comes in two flavors: Independent BSS, ad-hoc with no APs and Infrastructure BSS, includes both APs and stations.

C) Extended Service Set: This is a set of two or more BSSs sharing same network name, security credentials and also, providing translation between 802.3 and 802.11 .

D) Wireless Backbone: This binds up the various pieces of network, hence providing a path for information exchange between different LANs or sub networks.

Instead, there are some problems that are concerned with the wireless network. Out of which few of them can be as stated below:

a) The Hidden Terminal Problem: This occurs when a node is visible from a wireless AP, but not from other nodes communicating with that AP. The problem can be observed easily for radius $>50 \mathrm{~m}$. Fig. 1 represents the hidden terminal architecture.

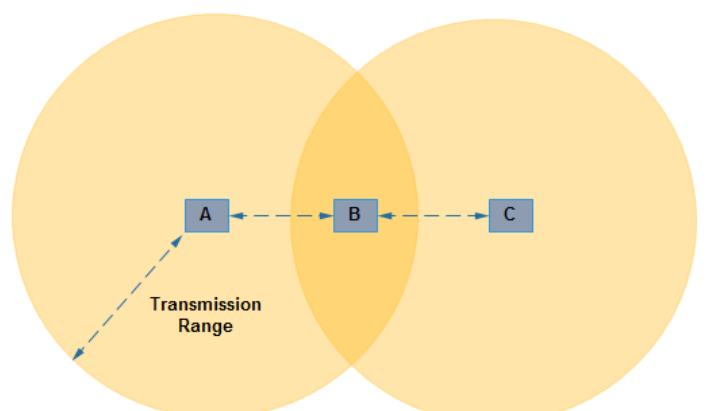

Figure 1: The Hidden Terminal Problem 
b) RTS/CTS Mechanism: It is one of the optimal mechanisms that help to reduce frame collisions. This includes the Ack concept, but does not solve the exposed node problem. Fig.2 depicts the underlined RTS/CTS mechanism.

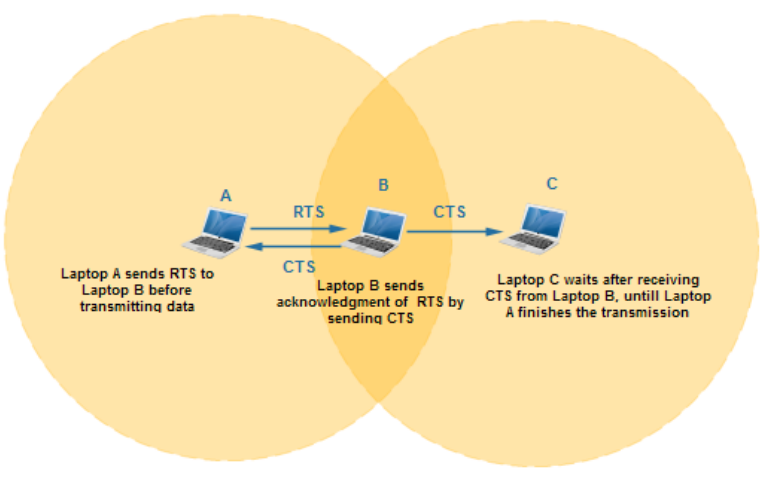

Figure 2: RTS/CTS Mechanism

c) Quality of Service: Measuring quantitatively the Quality of Service (QoS) [7] related to aspects of network services such as error rates, bandwidth, throughput, transmission delay, availability, etc.

The wireless technology is commonly used in radio. It encompasses different types of fixed, mobile and portable applications, including two-way radios, cellular phones, Personal Digital Assistance (PDA) and wireless networking.

\section{USEFUL WORK}

A WLAN is a wireless computer network that links up two or more devices using a wireless distribution method within a limited range such as a home, school, computer laboratory, or office building. This gives users the ability to move around in a particular coverage area and to easy access to information they want to. This becomes more popular in home as they are easy to install and use, also they may be made as free to employees for the organization.

Wireless network operates using radio frequency technology. The cornerstone of the wireless network is a device named AP [8]. The primary job of an AP is to broadcast a wireless signal that computers can detect and "tune" into. In order to connect to an AP and join a wireless network, computers must be equipped with wireless network adapters.

The IEEE802.11 has two basic modes of operation: ad-hoc mode and infrastructure mode. In ad-hoc mode, mobile units transmit directly peer-to-peer. In infrastructure mode, mobile units communicate through an AP that serves as a bridge to other networks (such as Internet or LAN).

A) Peer-to-Peer: Here, stations communicate only Peer-ToPeer (P2P). This is accomplished using independent Basic Service Set (BSS). Wireless devices within range of each other can discover and communicate directly without involving central APs. If the signal strength cannot be read accurately then this mode can lead to the hidden terminal problem.

B) Infrastructure: Ethernet (wired) connection is made onto the AP. A bridge can be used to connect with the network. A remote base station accepts connections from wireless clients and passes them to relay or main stations.
WLAN are increasingly popular. Groups from fast food restaurants to libraries offer the Internet over the air for free, and the equipment to set one up in a home or office is increasingly becoming cheaper. The following displayed are few advantages of using a WLAN:

a. Convenience: Connecting to them is as simple as logging onto a website.

b. Expandability: It is cheaper and less complicated, giving people with a lot of area to offer connections to a simple way to do so.

c. Availability of high reliability VoIP network services.

\section{OPNET SIMULATION}

For the sake of simulation, we have setup a wireless local area network that accomplished mobility on the TCP performance. Also, it is examined how the hidden terminal problem is avoided by using Request to Send (RTS) and Clear to Send (CTS) frames.

The key problem is to handle the mobile stations in the WLANs. IEEE802.11 utilizes the RTS and CTS frames in various circumstances further in order to minimize the collisions. Duration information in RTS/CTS frames are used to set in certain manner within the reception range, so as to solve the hidden sender because any station that sees the CTS frame knows that it is close to the receiver and, therefore, cannot transmit for the period of time indicated.

In simulation, a WLAN set up with mobile workstations and in certain aspects, the destinations are also defined to all the stations configured. It is found to study the effect of mobility onto the mobile node. Also it will help in computing the minimized hidden node problem induced by mobility in WLANs.

\section{A) First Scenario: Simple DCF Mode}

In this scenario, WLAN is created 20 stations are made to be placed establishing wireless environment. These stations are made to operate in a DCF based environment. The threshold is being shared in between all the stations.

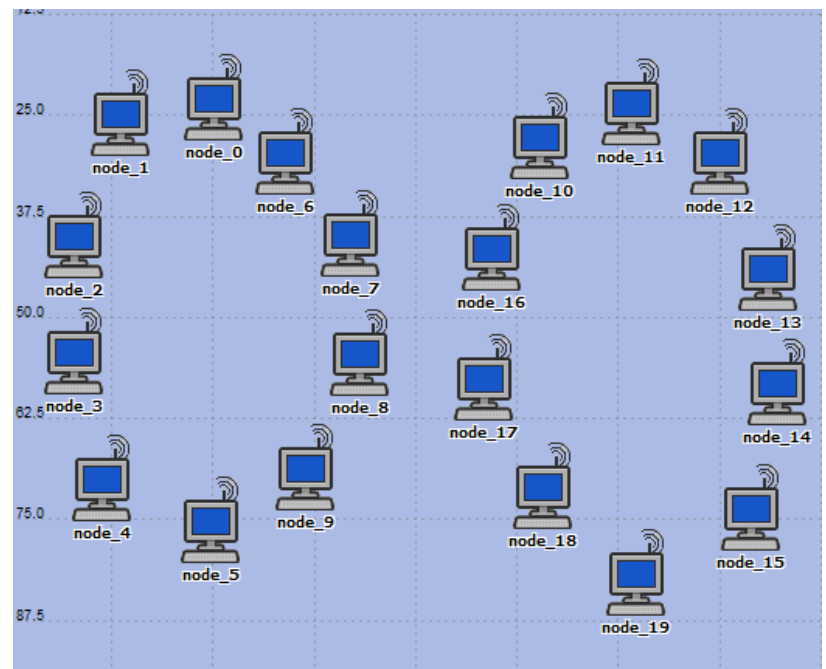

Figure 3: Stations communicating in DCF mode with no Fragments. 


\section{B) Second Scenario: DCF Mode with Fragmentation}

In this scenario, WLAN is created with 20 wireless stations that are being provided with the functionality of DCF environment. The threshold [9] is being divided into smaller fragments.

Under the fragmentation mechanism, the data packet received from a higher layer with a size greater than the threshold is being divided into smaller fragments. Fig. 3 represents that stations are communicating in DCF mode with Fragmentation.

\section{C) Third Scenario: Enhanced DCF with PCF Mechanism}

The PCF functionality in the first scenario (simple DCF mode), in a no fragmentation environment, is implemented here. Particular or even half of the stations are implemented using DCF and other half to the PCF technique. Fig. 3 represents that stations are communicating in enhanced DCF (PCF) [10] mode with no Fragmentation.

\section{D) Fourth Scenario: Enhanced DCF to PCF with Fragmentation}

Third scenario is enhanced here with the fragmentation functionality to better access the data packets flowing into the two specified modes (DCF and PCF). Fig. 3 represents that where stations are communicating in enhanced DCF (PCF) mode with Fragmentation.

\section{ANALYSIS OF SIMULATION}

After implementing and running the simulation for four scenarios defined above, on considering different aspects for which it justify which devices performs better under what conditions. The analysis for different scenarios on different aspects is as following:

1) Delay: Fig.4, Fig.5a and Fig.5b show the delay that represents an end-to-end delay for all the packets received by WLAN MACs of all the WLAN nodes in the configured network. This depicts the medium access delay at source $M A C$, reception of all the individual fragment, and transfer of frames through AP, if enabled.$$
\text { frames through AP, if enabled. }
$$

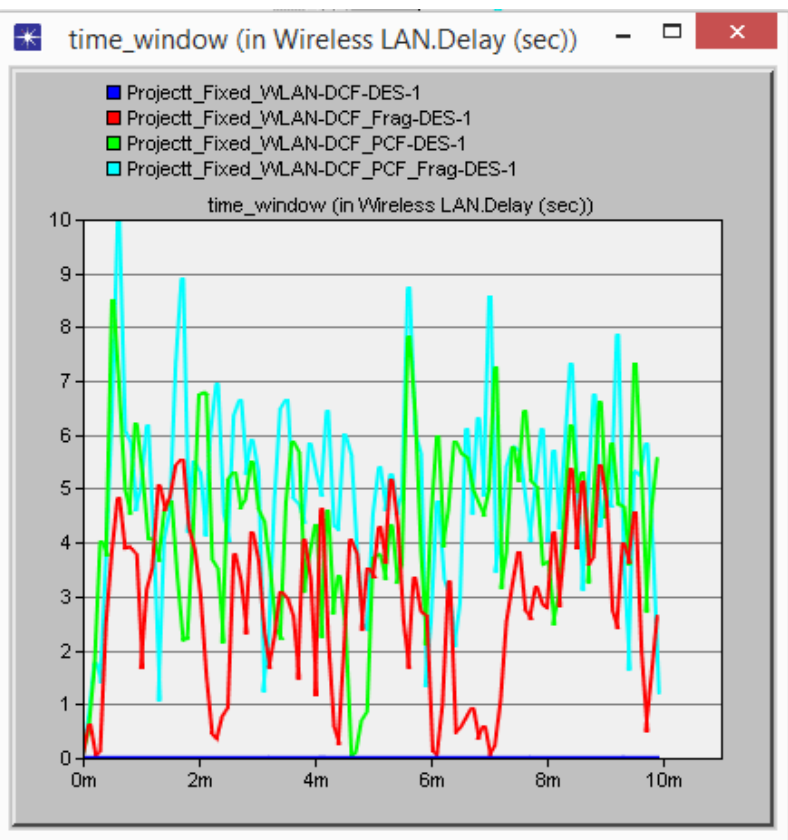

Figure 4: Delay in WLAN in all Scenarios.

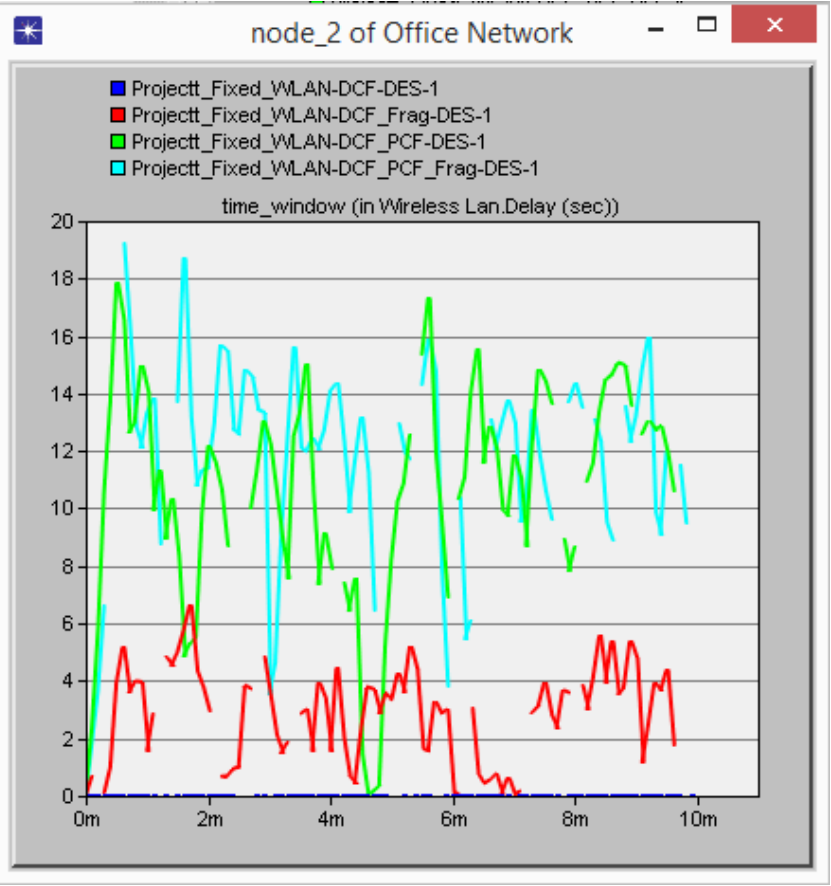

Figure 5a: Delay in node_2 in WLAN depending upon MACs.

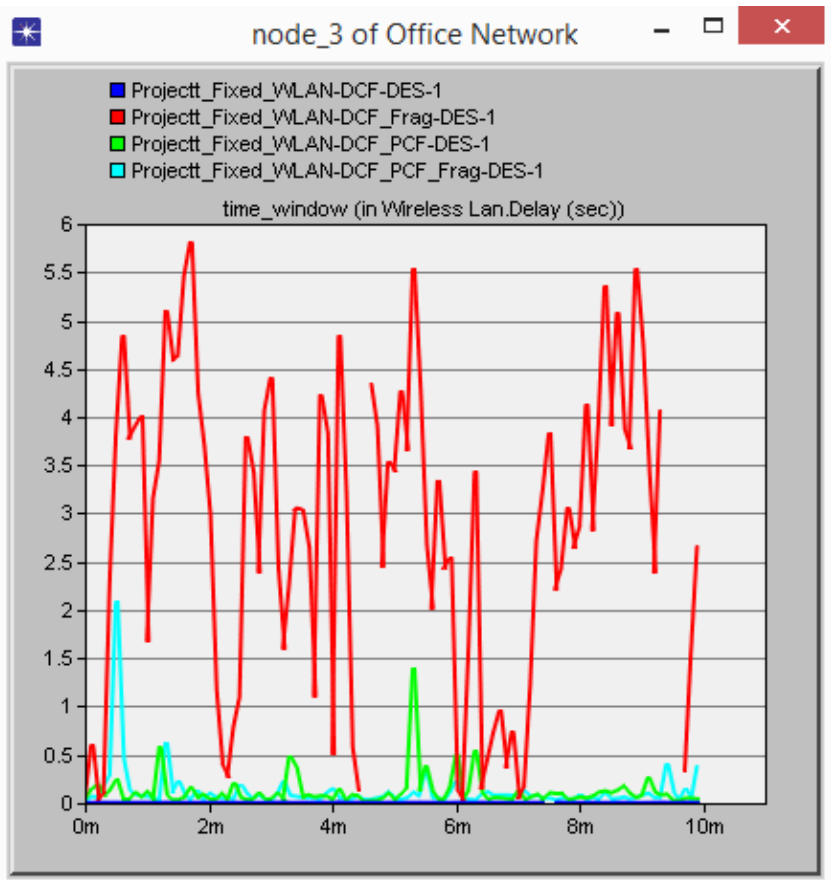

Figure 5b: Delay in node_3 in WLAN depending upon MACs

2) Load: Fig.6 represents the total load encountered in WLAN in all nodes in the network. The load can include the insufficient space left in the layer packet buffer of the MAC. 


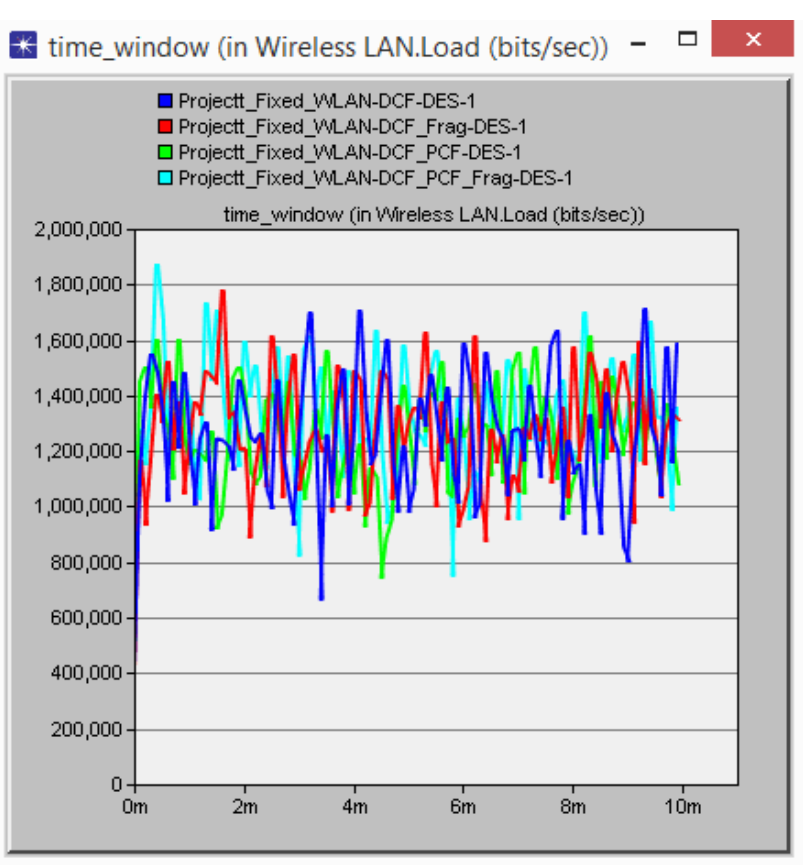

Figure 6: Load in WLAN in all scenarios.

3) Throughput: Fig.7 represents the total number of bits that are being forwarded from WLAN layer to the upper higher layers in all WLAN stations of the configured network.

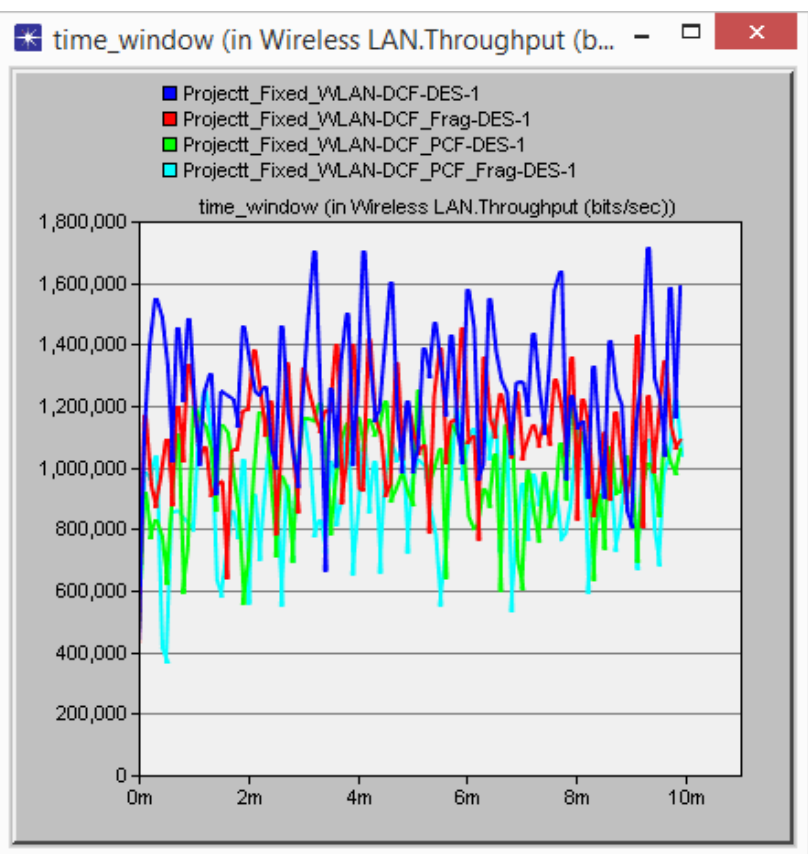

Figure 7: Throughput in WLAN in all scenarios.

4) Retransmission Attempts: Fig.8 represents the retransmissions attempted by the stations in the WLAN environment.

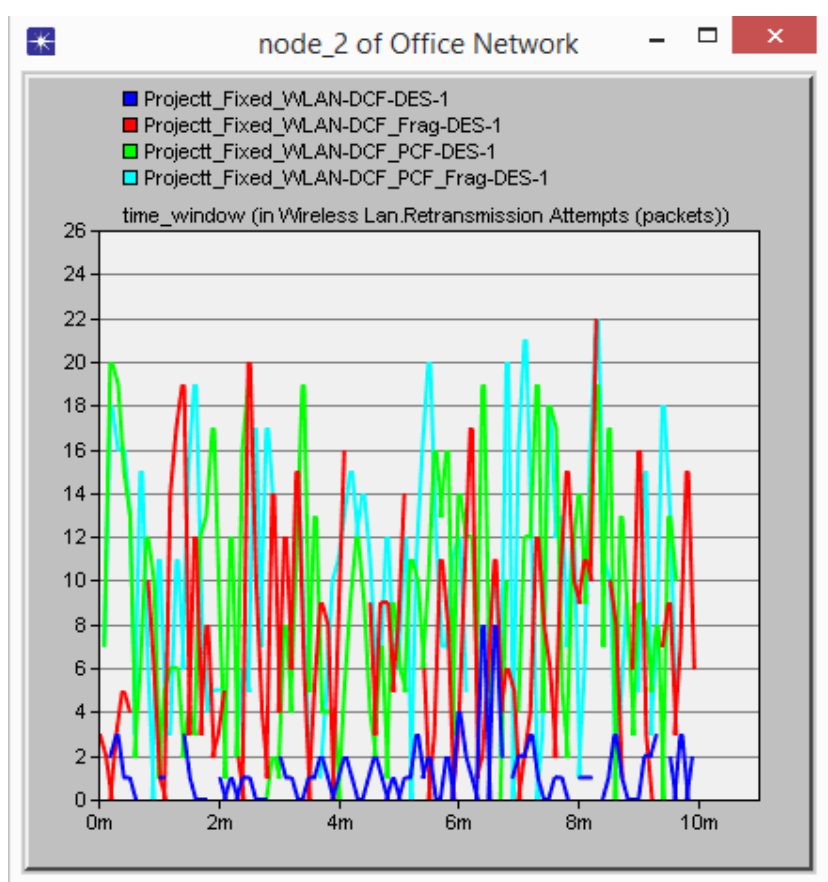

Figure 8a: Retransmission attempted by the node_2 in WLAN environment.

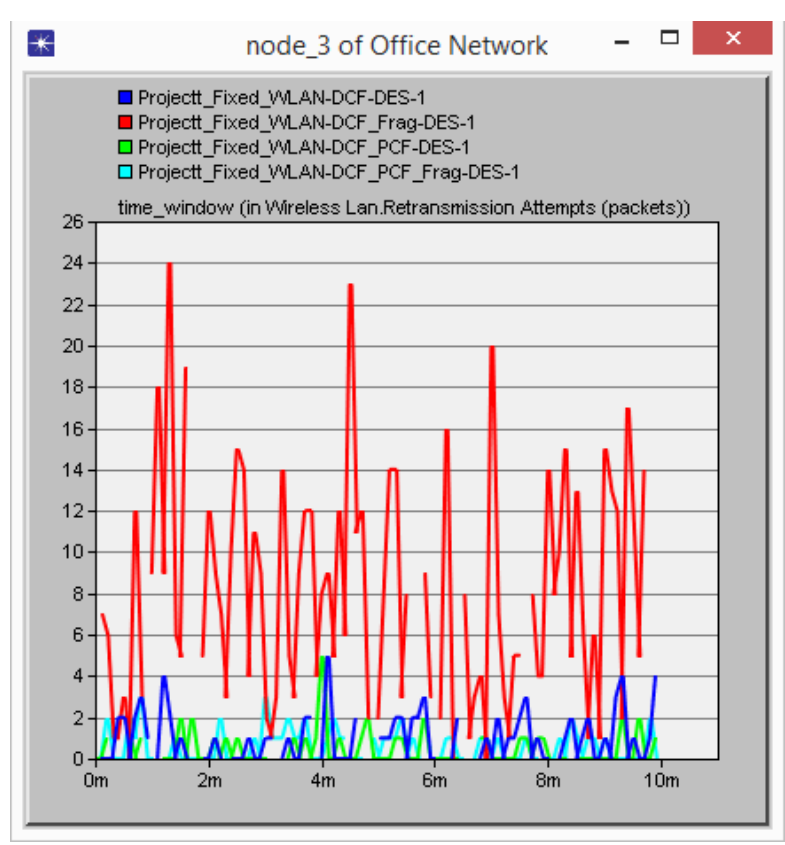

Figure 8b: Retransmission attempted by the node_ 3 in WLAN environment.

5) Media Access Delay: The delay that has taken place in considering to the fixed wireless stations in a WLAN environment. This is concerned with the shorter access delays that has taken place while transmission. Fig. 9 represents the medium access delay for all the accesses made. 
time_window (in Wireless LAN.Media Access Delay (sec))

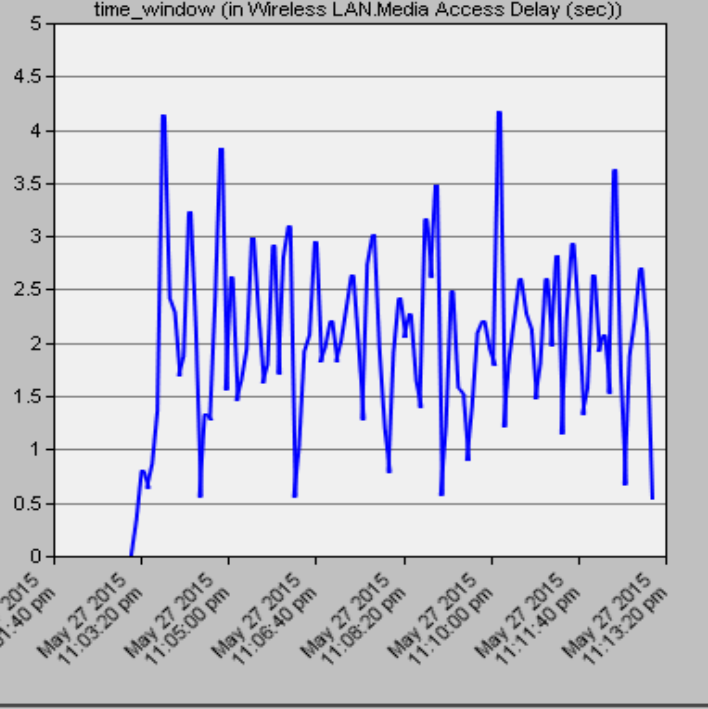

Figure 9: Media Access Delay in WLAN.

\section{CONCLUSION}

In this paper simulates the two protocols, i.e. DCF and PCF that are responsible to act into the WLAN environment. These two protocols use the medium access mechanism based on IEEE 802.11 standard. Fig.7 represents that the load factor is decreased up to an extent as the two classes minimizes the total waiting time required in transmission of data packets. The fragmentation of threshold provides with the best transmission results, and also through fragmentation of threshold the load at packet buffer has minimized.

Fig.7 clearly shows that the network is degraded using PCF mechanism that is a contention-free mechanism. This degradation can be reduced to an extent when implemented with the minimized polling structure at the AP that improves the throughput significantly.

\section{REFERENCES}

[1] "Wireless Ethernet Networking with 802.11b, an overview",

http://www.homenethelp.com/802.11b/index.asp
[2] "Wireless Local Area Networks (WLAN)", http://www.ictadvice.org.uk/downloads/wirelesslan_techn ical.doc

[3] Shweta Singh, Priyanka Mudgal, Priyadarshini Chaudhary, and Prof. Arun Kr. Tripathi: "Comparative analysis of Packet Loss in Extended LAN environment", International Journal of Computer Applications (IJCA), ISSN NO: 0975-8887, 20-April-2015.

[4] Eustathia Ziouva, Theodore Antonakopoulos. CSMA/CA performance under high traffic conditions: Throughput and delay analysis. Computer Communications Vol: 25 Issue: 3 ISSN: 0140-3664 Date: 02/2002 Pages: 313 - 32

[5] K. Xu, M. Gerla, and S. Bae, "How Effective is the IEEE 802.11 RTS/CTS Handshake in Ad Hoc Networks?" IEEE GLOBECOM'02, Vol. 1, pp. 72-76, November 2002.

[6] C. K. Toh, Ad Hoc Mobile Wireless Networks: Protocols and Systems, Prentice Hall, December 2001.

[7] R.K.Nadesh , D.Sumathy, M. B. BenjulaAnbu Malar, "Performance Analysis Of MANET (WLAN) Using Different Routing Protocols In Multi Service Environments-An Quantitative Study", Int. J. Advanced Networking and Applications Volume: 03, Issue: 02, Pages:1076-1079 (2011).

[8] J. Weinmuller, H. Woesner, J. Ebert, A. Wolisz, "Analyzing the RTS/CTS Mechanism in the DFWMAC Media Access Protocol for Wireless LANs," IFIP TC6 Workshop on Personal Wireless Communications, pp. 117-130, April 1995

[9] Rajan, R., Shipra, S. "WLAN Performance Improvisation by Fine Tuning IEEE 802.11 Parameters", International Journal of Computer Applications, April 2012.

[10] Wireless Central Coordinated Protocol (WiCCP), A Soft war Solution Of The Hidden Node Problem (http://patraswireless.net/software.html). 